\title{
Scale-free convection theory
}

\author{
Stefano Pasetto ${ }^{1}$, Cesare Chiosi ${ }^{2}$, Mark Cropper ${ }^{1}$ and Eva K. Grebel ${ }^{3}$ \\ ${ }^{1}$ Mullard Space Science Laboratory, University College London, Holmbury, St. Mary, \\ Dorking, Surrey RH5 6NT, United Kingdom \\ email: s.pasetto@ucl.ac.uk \\ ${ }^{2}$ Dept. of Physics \& Astronomy "Galileo Galilei", University of Padua, Vicolo \\ dell'Osservatorio, 5, 35141 Padova PD, Italy, \\ ${ }^{3}$ Astronomisches Rechen-Institut, Zentrum für Astronomie der Universität Heidelberg, \\ Mönchhofstr 12-14, 69120 Heidelberg, Germany
}

\begin{abstract}
Convection is one of the fundamental mechanisms to transport energy, e.g., in planetology, oceanography, as well as in astrophysics where stellar structure is customarily described by the mixing-length theory, which makes use of the mixing-length scale parameter to express the convective flux, velocity, and temperature gradients of the convective elements and stellar medium. The mixing-length scale is taken to be proportional to the local pressure scale height of the star, and the proportionality factor (the mixing-length parameter) must be determined by comparing the stellar models to some calibrator, usually the Sun. No strong arguments exist to claim that the mixing-length parameter is the same in all stars and all evolutionary phases. Because of this, all stellar models in the literature are hampered by this basic uncertainty. In a recent paper (Pasetto et al. 2014) we presented the first fully analytical scale-free theory of convection that does not require the mixing-length parameter. Our self-consistent analytical formulation of convection determines all the properties of convection as a function of the physical behaviour of the convective elements themselves and the surrounding medium (be it a star, an ocean, or a primordial planet). The new theory of convection is formulated starting from a conventional solution of the Navier-Stokes/Euler equations, i.e. the Bernoulli equation for a perfect fluid, but expressed in a non-inertial reference frame co-moving with the convective elements. In our formalism, the motion of convective cells inside convective-unstable layers is fully determined by a new system of equations for convection in a non-local and time dependent formalism. We obtained an analytical, non-local, time-dependent solution for the convective energy transport that does not depend on any free parameter. The predictions of the new theory in astrophysical environment are compared with those from the standard mixing-length paradigm in stars with exceptional results for atmosphere models of the Sun and all the stars in the Hertzsprung-Russell diagram.
\end{abstract}

Keywords. Sun: fundamental parameters, Sun: interior, stars: evolution, stars: fundamental parameters

\section{Results}

Despite its great importance, a satisfactory treatment of stellar convection in stars is still open to debate and a self-consistent treatment of the physics of convective energy transfer is still missing. In a recent paper, Pasetto et al. (2014) developed the first theory of stellar convection in which the solar properties are reproduced without making use of free parameters in great detail.

\section{Reference}

Pasetto, S., Chiosi, C., Cropper, M., \& Grebel, E. K., 2014, MNRAS, 445, 3592 